\title{
The combined approach to evaluation of mechanical behavior of tooth enamel in artificially induced caries lesions
}

\author{
Sergey Galkin ${ }^{1}$, Anna Levitskaya ${ }^{2}$, Olga Gileva ${ }^{2, *}$, Tatiana Libik ${ }^{2}$, and Larisa \\ Lomiashvili $^{3}$ \\ ${ }^{1}$ Perm National Research Polytechnic University, Department of Oil and Gas Geology, 614990, \\ Komsomolsky prospect, 29, Perm, Russian Federation \\ ${ }^{2}$ Perm State Medical University named after Academician E.A. Vagner, Department of Therapeutic \\ and Preclinic Dentistry, 614990, Petropavlovskaya st., 26, Perm, Russian Federation \\ ${ }^{3}$ Omsk State Medical University, Department of Therapeutic Dentistry, 644099, Lenina st., 12, Omsk, \\ Russian Federation
}

\begin{abstract}
Improved knowledge of caries initiation and progression on the base of modern mechanobiology could facilitate effective preventing and therapeutic strategies. Not all of previously developed experimental models of artificial caries comply with required standards. The aim of the study was to investigate microstructural and mechanical properties of 12 artificial caries lesions, induced on human intact premolars according to original protocol. The tooth specimens were subjected to imaging by X-ray CT system Nikon Metrology XT H 225, with further morphometric analysis of lesion geometry, depth, surface layer thickness and mineral density in the areas of interest. Mechanical characteristics (penetration depth, microhardness, elastic modulus) in enamel lesion, perifocal intact enamel and remote zones were obtained using Micro CombiTester system. Clinically all lesions fully corresponded to ICDAS -2 code. According to $\mu \mathrm{CT}$ data, $\mathrm{LD}_{\mu \mathrm{CT}}$ of the lesions ranged from 346.4 to $531 \mu \mathrm{m}$, mineral density range from $2.03 \pm 0.15 \mathrm{~g} / \mathrm{cm}^{3}$ in transparent zone to $1.05 \pm 0.08 \mathrm{~g} / \mathrm{cm}^{3}$ in the surface zone of the lesion. Microhardness of demineralized enamel lesion more than 5 times reduced in comparison with perifocal and remote intact enamel. Nanoindentation and $\mu \mathrm{CT}$ data are corresponds to those presenting on the base of other classical protocols of artificial caries formation and close to the typical mechanical characteristics of enamel caries in vivo.
\end{abstract}

\section{Introduction}

Aging and the related changes in mechanical properties of the hard dental tissues are becoming increasingly important for modern dentistry and gerontology $[1,2]$. Throughout lifetime the hard tissues of the teeth (enamel, dentin) are constantly exposed to bacterial

\footnotetext{
* Corresponding author: o.s.gileva@yandex.ru
} 
load, numerous chemical and physical impact, which results in their miscellaneous damage such as enamel demineralization, abrasion, attrition-corrosion, crack and cavity formation. A great body of fundamental research focuses on the pattern of mechanical behaviour of the enamel in healthy aging, appeared in increase of its hardness and elastic modulus in parallel with the decrease of its permeability and side-specific thickness [3, 4]. A comprehensive study of the changes in physical properties of human tooth enamel and dentin in elderly is of a great importance for the prevention of tooth pathological aging, as well as for treatment and prophylaxis of conventional carious diseases and its age-dependent type (root caries) [5]. The most common enamel lesions are observed in dental caries - multifactorial, oral biofilm-mediated disease in which a progressive loss of a mineral component (demineralization) of enamel occurs under the action of acid-producing bacteria. Enamel demineralization, or white spot lesion, is considered to be an incipient stage of carious disease. Well-timed diagnostic, non-invasive or mini-invasive treatment and prevention of the white spot lesion are among top-trends of current cariology [6].

Improved knowledge of the dental caries initiation and progression on the base of principles of modern mechanobiology could facilitate effective caries preventive and therapeutic approaches. The earliest stages of caries (enamel demineralization) have been fully studied at macro-, meso- and micro/nanoscopic levels by macroscopic examination, stereo- or light microscopy, scanning or transmitted electron microscopy, atomic force microscopy, microradiography, micro/macro-indentation, X-ray imaging techniques, including conventional X-ray computer and microfocus X-ray computer tomography optical-coherent tomography [7-14]. Significant information about the pattern of caries development is provided by mathematical modelling methods $[15,16]$. There is significant knowledge on changes in the surface topology, micro / nanostructure, mechanical properties and chemical homeostasis of affected (carious, eroded, bleached, etc.) tooth enamel [17-20].

With all the variety of experimental methods for multiscale analysis of the structure and mechanical properties of healthy and carious enamel, the value of many of them is limited by one or the other degree of invasiveness, difficulties in receiving of quantitative data, the need for expensive technical equipment and especially for the adaptation of its capabilities to analyse such a small-size objects as teeth and their segments.

Modern imaging technologies / X-ray CT and created on its basics X-ray MicroCT allow to conduct non-invasive visualization of internal tooth structures in $3 \mathrm{D}$, avoiding most of above-mentioned constraints. X-ray computer (microcomputer) qualitative and quantitative analysis of the enamel structure is carried out in relatively short time, doesn't require special sample preparation or its destruction. X-ray techniques allow to characterize the geometrical parameters of the enamel (phase shares, porosity, pore size and their statistical distribution: its thickness and mineral density (via densitometry) at different depth and in different zones of the lesion, and to monitor their changes in the course of a certain treatment.

The accuracy and sensitivity of X-ray microtomographic study of the internal structure micromorphology tooth enamel and dentin are comparable to that of histological or microradiographic techniques, considered as the "gold standard methods", although required the damage of the tooth species [21,22].

A variety of laboratory X-ray CT or MicroCT scanners have been developed, not all of them are well adapted to the analysis of the internal structure and mineral density of such small-size samples as human tooth enamel. We have not seen enough information on the application of X-ray CT system Nikon Metrology XTH 225 for the needs of basic dentistry. It is disclosed in one-off publication on the structural characterization of in vitro affected tooth enamel [23]. 
The value of the information on the microstructure and mineral density of enamel lesion, provided by microtomography, increases when the main mechanical characteristics of this biomaterial - hardness and elastic modulus are obtained and analyzed in parallel. The combination of these two methods might prove useful in evaluating the reliability of a new in vitro-models of enamel demineralization, traditionally used for testing different caries-preventive or anticaries dental products.

Microhardness data are rightly considered as a precise indicator of the degree of tooth enamel demineralization due to the high correlation between its cross-sectional microhardness and \% vol mineral determined by transversal microradiography, that is essential method to determine the amount of mineral gain or loss in subsurface caries lesion in vitro [24]. Modern nanoindentation systems enables investigations of microhardness and elastic modulus under different loading conditions based on load displacement data of indentations on submicron scale $[25,26]$.

Caries basic research usually ultimately aimed at testing new treatment or preventive technologies in the phase between lab testing and clinical trials. Such studies usually conducted using in vitro-models of artificial caries lesion. There are a large diversity of models (protocols of formation) of in vitro enamel demineralization, but not all of them are able to simulate the complex biological process involved in caries, and, thus, are not comply with all required standards [27, 28].

The aim of the study is to investigate structural and mechanical characteristics of artificial caries lesions, induced according to original protocol of in vitro demineralization, using microtomographic and nanoindentation techniques.

\section{Materials and Methods}

\subsection{Experimental design, preparation of samples, and protocol of artificial caries lesion formation}

This in vitro study was approved by the Institutional Research and Ethic Committee of PSMU named after Academician E.A.Vagner and executed at the support of RFBR (grant No. 17-48-590562-r_ural). For the in situ experiment twelve intact premolars obtained from orthodontic patients (18-25 y.o.) of participating dental clinics within Perm and Omsk cities signing an informed consent were selected. All clinical and experimental procedures were carried out according relevant guidelines and regulations. The samples of extracted teeth have been initially treated, using special atraumatic mechanical and chemical protocol.

\subsubsection{Protocol of artificial caries lesion formation}

The foci of tooth enamel demineralization were modeled on the vestibular surface of the crown (Fig. 1) using the original lab equipment, tools and solutions: device for in vitro caries formation by enamel window technique (the patent of RF for useful model №172561, 16.12.2016), separator for storage of tooth biomaterials (the patent of RF for useful model №171409, 16.12.2016), demineralizing gel of the original composition [3 g Praestol 2510 (polyacrylamide with a molecular weight of approximately 14 million units], $8 \mathrm{ml}$ of $80 \%$ lactic acid, $0.4 \mathrm{~g}$ of calcium dihydrogen phosphate, the sodium hydroxide solution $1 \mathrm{~g}$ in $10 \mathrm{ml}$ of water (add a drip until the $\mathrm{pH}$ of the solution 4,5), distilled water up to a volume of $1000 \mathrm{ml}$ ) used in a special time-temperature regimen in accordance with the efficiency suggestion of PSMU No. 2757, 17.05.2018.

Micro-CT studies were carried out in the laboratory of petrophysics of Perm National Research Polytechnic University. Physical and technical characteristics of Nikon Metrology XT H225 X-ray computer tomograph are as follows: voltage $(\mathrm{kV})-140$, current $(\mathrm{mA})-$ 65 , filter copper $(\mathrm{mm})-0.25$, focal spot size $(\mathrm{mm})-0.003$, the dimensions of the detector 
$(\mathrm{mm})-200 \times 250$, detector resolution (pixel) $-1408 \times 1888$, resolution in pixel $(\mathrm{mm})-$ 0.0184 , frame rate $(\mathrm{Hz})-3.5$, the magnification $-\times 12$. Microtomographic analysis included $3 \mathrm{D}$ reconstruction of the tooth hard tissues, including enamel zone, the analysis of their volumetric features, the state of tissues structure, review analysis of axial 2D slices crowns. Emphasis was placed on morphometric analysis of the enamel lesion, its geometrical peculiarities (the shape, length by width $(\mathrm{mm})$, lesion depth $\left(\mathrm{LD}_{\mu \mathrm{CT}}, \mu\right)$, the surface layer thickness $\left(\mathrm{LSL}_{\mu \mathrm{CT}}, \mu\right)$, as well as on the mineral density $\left(\mathrm{g} / \mathrm{cm}^{3}\right)$ in the areas of interest by reference densitometry, and the mineral loss $(\Delta \mathrm{Z}, \%)$ as a percentage of healthy enamel density. For mineral density analysis, a series (5) of mineral reference disks were prepared.

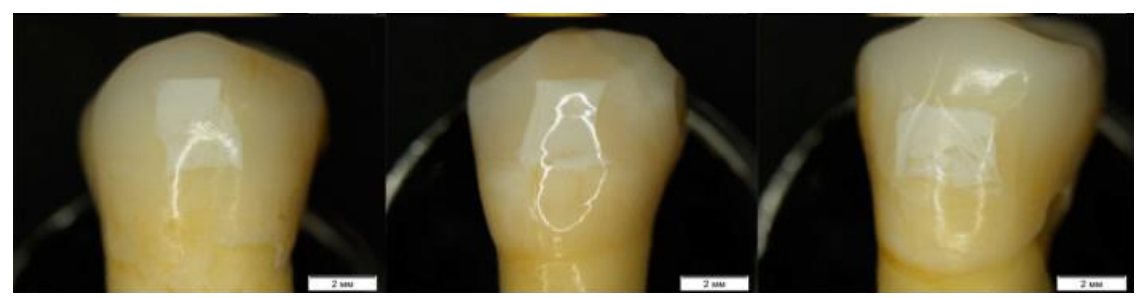

Fig. 1. Digital images of teeth with artificially induced foci of enamel demineralization (K02.0 caries of enamel / white spot lesion; code 2 according to ICDAS)

Mechanical characterization of the intact and demineralized enamel was obtained using a high-precision kinetic indentation method, using the Micro CombiTester $\left(\mathrm{MCT}^{3}, \mathrm{CSM}\right.$ Instruments SA) machine (diapason of load $(\mathrm{N})-0,05-30$; load resolution $(\mu \mathrm{N})-0,3$; depth range $(\mu \mathrm{m})-600$; depth resolution $(\mathrm{nm})-0,03$; load speed $(\mathrm{mm} / \mathrm{min})-0,1-300$; platform $\mathrm{XY}-120 \mathrm{~mm} \times 20 \mathrm{~mm}$; resolution $\mathrm{XY}-0,25 \mu \mathrm{m}$; videoscope resolution $-\times 200, \times 800$, $\times 2000$. MCT-indentor equipped with video microscope $(768+582)$, diamond Vicker's pyramid ( $\alpha=136 \pm 0,2)$. The tests were performed according to International ISO 14577 , that specifies the indentation of the material with the registration of the load on the indentor, the movement of the indentor, and indentation time during elastic and plastic deformation of the material. Indentation tests were made in the central part of the area of enamel demineralization, perifocal visually intact enamel and distant intact sound enamel areas, respectively. The indentation was performed in increments of 10 to $30 \mu \mathrm{m}$ in both (X, Y) directions. A load of $20 \mu \mathrm{N}$ was given for all probes, time of loading and unloading reached $270 \mathrm{~s}$. Each of the studied zones of the enamel was indented 20 times. Indentations were made from the outer surface at $10 \mu \mathrm{m}$ up to $60 \mu \mathrm{m}$. Finally, the mean values at all examined points at each distance were calculated. The enamel hardness (HIT, GPa) and elastic modulus (EIT, GPa) were calculated using Oliver and Pharr method that is considered as a gold standard for indentation data analysis.

Both mechanical indicators were analyzed in parallel with the calculation of the depth of indentor penetration $(\mathrm{Pd}, \mu \mathrm{m})$. Cross sections of teeth (Fig. 2a) were prepared for testing, after processing in grinding machine they were washed in an ultrasonic bath. Optical microscopic images of demineralized enamel with indentor's traces presented on Fig. $2 b$. 


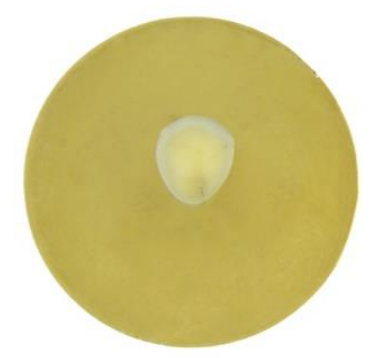

Fig. 2a. Cross section of the tooth prepared for microindentation

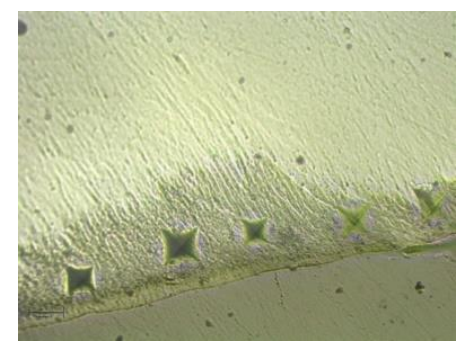

Fig. 2b. Optical microscopic images of demineralized enamel with traces of Berkovich tip indentor

\subsection{Statistical Analysis}

Statistical evaluation of the data was performed using Statistica 13 software with calculation of the mean and standard mean-square error of the mean. Reliability of differences was calculated by U Mann-Whitney criterion. P-values were derived and values of $<0.05$ were considered statistically significant.

\section{Results and Discussion}

Clinical signs (colour, texture, geometry) of artificially created caries indicated the appearance of typical areas of enamel demineralization, mainly opaque - white in colour and square in shape, on the vestibular surface of all 12 tooth samples. Colour and texture characteristics of the white carious spots fully corresponded to code 2 ICDAS, that justified the high sensitivity of created caries experimental model from clinical point of view. 3D images of dental crowns with artificially induced enamel demineralization indicated conformity of internal and external anatomy of teeth and their hard tissues with the structure of the premolars of the upper or lower jaws. The zones of enamel demineralization was painted with red to the depth of a third of the enamel. Morphometric data of 30 axial $2 \mathrm{D}$ sections of the crown pointed out shape, length in width, maximum depth of demineralized area, thickness of the enamel through the affected zone at its maximum depth (Fig. 3). 


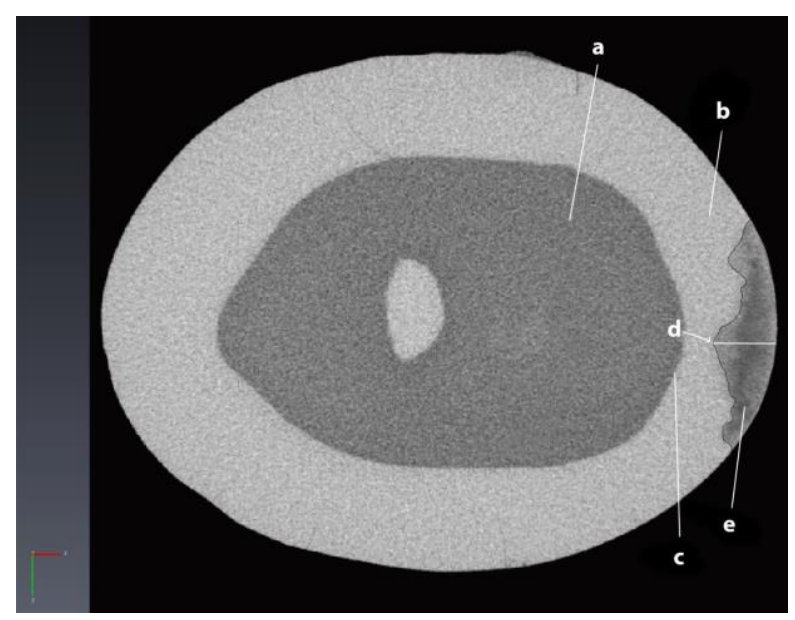

Fig. 3. Axial 2D-cut of the crown 3.4 tooth, passing through the demineralization zone at its maximum depth: $\mathrm{a}$ - dentin; $\mathrm{b}$ - intact enamel; $\mathrm{c}$ - enamel-dentine border; $\mathrm{e}$ - the center of enamel demineralization with the point of maximum depth (d)

The vast majority of created enamel lesions had a triangular shape.

$\mathrm{LD}_{\mu \mathrm{CT}}$ ranged from 346,4 to $531 \mu \mathrm{m}$ with an average value of the maximum depth $423.8 \pm 107.4 \mu \mathrm{m}$ which well correlates with data obtained in the other in vitro models of enamel caries according to Cochrane et al. (2012) - 275 to $846 \mu \mathrm{m}$ [9] and Espigares et al. $-402 \pm 163 \mu \mathrm{m}$ [29]. It was crucial that these indicators in artificially induced lesions correspond to the depth of enamel damage in natural white spot lesion according to Hamba et al. $-150-950 \mu \mathrm{m}[30]$.

In $\mu \mathrm{CT}$, the demineralized areas appeared radiolucent. The intact surface layer was well contoured in all samples. Artificial enamel lesions appeared as dark zones below the tooth surface. The thickness of the intact zone of enamel, laying under the foci of lesion to the enamel-dentin border (EDB) range of $568.0 \pm 107.3 \mu \mathrm{m}$; its mineral density was $2.76 \pm$ $0.07 \mathrm{~g} / \mathrm{cm}^{3}$, that correspond to the data of intact enamel.

According to microtomographic data, the entire locus of enamel demineralization was mapped into different zones (surface zone, body of lesion, dark zone, transparent zone). The transparent zone of the enamel lesion had significantly large thickness $(173.5 \pm 73.4$ $\mu \mathrm{m})$, while was almost 4 times thinner $(43.1 \pm 31.2 \mu \mathrm{m})$ the thickness of the "body" of the lesion and dark zone $-60.3 \pm 35.1 \mu \mathrm{m}$, and $86.6 \pm 37.2 \mu \mathrm{m}$, respectively. The most significant changes in mineral density was recorded in dark zones of caries lesions ( $\max -$ $1.64 \pm 0.11 \mathrm{~g} / \mathrm{cm}^{3} ; \mathrm{min}-1.29 \pm 0.23 \mathrm{~g} / \mathrm{cm}^{3}$, with average value $\left.1.47 \pm 0.17 \mathrm{~g} / \mathrm{cm}^{3}\right) ; 53.1 \%$ relative to the mineral saturation of healthy enamel.

The mineral density indicators in the central part of the lesion (body) ranged from 1.64 $\pm 0.11 \mathrm{~g} / \mathrm{cm}^{3}$ to $2.07 \pm 0.12 \mathrm{~g} / \mathrm{cm}^{3}$ (average value $1.86 \pm 0.17 \mathrm{~g} / \mathrm{cm}^{3}$ ) was significantly reduced $(67.2 \%)$ in comparison with the surface zone. Indices were in consistent with data reported for real-life (in vivo) carious lesions of the human enamel [Shahmoradi, 2016]. Mineral density of the surface zone of the lesion was $1.56 \pm 0.12 \mathrm{~g} / \mathrm{cm}^{3}$ on average (max $2.07 \pm 0.12 \mathrm{~g} / \mathrm{cm}^{3} ; \min -1.05 \pm 0.08 \mathrm{~g} / \mathrm{cm}^{3} ; 56.5 \%$ ) relatively intact human enamel.

According to experts [3] mechanical properties of healthy tooth enamel reflected in the hardness parameters range from approximately $3 \mathrm{GPa}$ to $66 \mathrm{GPa}$ and elastic modulus varies from $70 \mathrm{GPa}$ to nearly $120 \mathrm{GPa}$ according to area and depth of enamel indentation, age of the patient, and etc. Our results of testing mechanical properties of in vitro demineralized and intact dental enamel presented in the Table 1. 
Table 1. Penetration depth (Pd), microhardness (HIT, GPa), elastic modulus (EIT, GPa) in the areas of the tooth surface: the central part of the demineralized zone; the perifocal zone of intact enamel; the area of intact enamel remote from the lesion.

\begin{tabular}{|c|c|c|c|}
\hline Tested area & $\begin{array}{c}\text { Pd, } \mu \mathrm{m} \\
\text { Mean } \pm \text { SD }\end{array}$ & $\begin{array}{c}\text { HIT, GPa } \\
\text { Mean } \pm \text { SD }\end{array}$ & $\begin{array}{c}\text { EIT, GPa } \\
\text { Mean } \pm \text { SD }\end{array}$ \\
\hline Central, demineralized & $0.62 \pm 0.04^{*, * *}$ & $0.956 \pm 0.15^{*, * *}$ & $10.345 \pm 1.35^{*, * *}$ \\
\hline Perifocal, visually intact & $0.23 \pm 0.01^{*, * * *}$ & $5.006 \pm 0.54^{*}$ & $83.812 \pm 14.4^{*}$ \\
\hline Intact, far from the lesion & $0.19 \pm 0.01^{* *, * * *}$ & $5.860 \pm 0.25^{* *}$ & $85.427 \pm 6.6^{* *}$ \\
\hline
\end{tabular}

$*_{\mathrm{p}}$-value $<0.05$ between central and perifocal areas

$* * \mathrm{p}$-value $<0.05$ between central and intact areas

$* * *$ p-value $<0.05$ between perifocal and intact areas

It is predictable, that penetration depth was maximum $(0.62 \pm 0.04 \mu \mathrm{m})$ in the central area of demineralization zone, and was significantly $(\mathrm{p}<0,001)$ higher than in the adjacent and in distant areas of intact enamel $(0.23 \pm 0.001 \mu \mathrm{m}, 0.19 \pm 0.01 \mu \mathrm{m}$, respectively). Penetration depth in perifocal, visually intact areas of the enamel $(0.23 \pm 0.001 \mu \mathrm{m})$ was significantly higher $(\mathrm{p}<0.05)$ than in its remote zones.

The microhardness of the central zone of artificial caries $(0.956 \pm 0.15 \mathrm{GPa})$ was considerably $(\mathrm{p}<0.001)$ by more than 5 times reduced in comparison with both observed zones of healthy enamel: perifocal enamel $(5.006 \pm 0.54 \mathrm{GPa})$ or remote intact enamel $(5.86 \pm 0.25 \mathrm{GPa})$. The elasticity indicators of the central demineralized zone were significantly $(\mathrm{p}<0.001)$ decreased compare with similar indicator in healthy enamel - up to $10.345 \pm 1.35 \mathrm{GPa}$ in average. Both mechanical parameters in all indented zones of intact enamel (perifocally or distantly located) did not differ significantly, although they showed a downward trends in the areas closer to focus of lesion. The data on depth-related mechanical properties of artificial caries lesions are generally the same as those obtained on the other artificial caries in vitro-models [26]. Mineral density-depth profile of intact zones of enamel around focus of demineralization described in the study, corresponds to those presenting in available research literature in relation to healthy tooth enamel in middle-aged people.

\section{Conclusion}

Based on the microtomographic and $\mu \mathrm{CT}$ data of the mechanical properties, mineral density and microstructure of artificially-induced caries lesions we can conclude that our original protocol of artificial caries formation is reliable to reproduce enamel demineralization in vitro and, thus, can be used for basic dental research as well as for testing different caries preventive or anticaries products. The X-ray CT system Nikon Metrology XT H 225 can be successfully used for needs of basic dentistry, been well adapted for analyzing of microstructure and mineral density of such small-size samples as human tooth enamel. The data on depth-related mechanical properties obtained by nanoindentation technique stress the importance of monitoring the state of intact enamel, located perifocally to the zone of demineralization as the indices of penetration depth, microhardness, and elastic modulus of this area reduced in comparison with the value of intact enamel. 


\section{References}

1. D.D. Arola, S. Gao, H. Zhang, R. Masri, Dent. Clin. North Am., 61(4), 651-668 (2017). doi:10.1016/j.cden.2017.05.001

2. A.A. Kunin, A.Y. Evdokimova, N.S. Moiseeva, EPMA J., 6(1), 3 (2015). doi:10.1186/s13167-014-0025-8

3. S, Park, D.H. Wang, D. Zhang, E. Romberg, D. Arola, J. Mater. Sci. Mater. Med., 19(6), 2317-2324 (2008). doi:10.1007/s10856-007-3340-y

4. T.S. Carvalho, A. Lussi, J. Oral Rehabil., 44(4), 291-298 (2017). doi:10.1111/joor.12474

5. E.V. Serebrennikova, O.S. Gileva, Perm Medical Journal, 26(2), 129-134 (2009)

6. O.S. Gileva, Perm Medical Journal, 35(6), 61-72 (2018)

7. A.Y. Belyaev, O.S. Gileva, M.A. Muravieva, A.L. Svistkov, A.P. Skachkov, Russian Journal of Biomechanics, 16(3), 57-64 (2012)

8. D.V. Zaytsev, S.S. Grigoriev, O.V. Antonova, P.E. Panfilov, Russian Metallurgy (Metally), 6, 37-44 (2011)

9. N.J. Cochrane, P. Anderson, G.R. Davis, G.G. Adams, M.A. Stacey, E.C. Reynolds, Journal of Dental Research, 91(2), 185-191 (2012)

10. B.M. Ozgul, K. Orhan, F.T. Oz, Journal of Oral Science, 57(3), 177-183 (2015)

11. M. Shahmoradi, M.V. Swain, J. Dent., 46, 23-29 (2016).

12. M. Skucha-Nowak, A. Machorowska-Pieniążek, M. Tanasiewicz, Advances in Clinical and Experimental Medicine, 1.25(5), 961-969 (2016). doi: 10.17219/acem/63007

13. M.J. Kim, S.H. Lee, N.Y. Lee, I.H. Lee, Arch. Oral Biol., 58(2), 160-166 (2013). doi:10.1016/j.archoralbio.2012.06.015

14. E.B. Gurdogan, D. Ozdemir-Ozenen, N. Sandalli, J. Esthet. Restor. Dent., 29(3), 201-208 (2017). doi:10.1111/jerd.12279

15. O.S. Gileva, M.A. Muraveva, N.I. Simakina, A.K. Sokolov, V.N. Terpugov, Vestnik Permskogo universiteta. Seria: Matematika. Mechanika. Informatika, 2, 20-25 (2012)

16. R.I. Izumov, A.L. Zuev, S.V. Rusakov, O.S. Gileva, M.A. Muraveva, Key Engineering Materials, 592-593, 362-365 (2014).

17. I.A. Morozov, A.L. Svistkov, O.S. Gileva, E.S. Erofeeva, Russian Journal of Biomechanics, 14(1), 56-64 (2010)

18. I.A. Morozov, A.Y. Belyaev, R.I. Izyumov, E.S. Erofeeva, O.S. Gileva, Inorganic Materials : Applied Research, 4(1), 71-76 (2012)

19. E.S. Erofeeva, E.A. Lyapunova, V.A. Oborin, O.S. Gileva, O.B. Naimark, Russian Journal of Biomechanics, 14(2), 47-55 (2010)

20. A.Y. Belyaev, A.L. Zuev, O.S. Gileva, M.A. Muraveva, Key Engineering Materials, 592-593, 358-361 (2014)

21. R. Chalas, K. Szlazak, I. Wojcik-Checinska, J. Jaroszewicz, R. Molak, K. Czechowicz, S. Paris, W. Swieszkowski, K.J. Kurzydlowski, Folia morphologica, 76(2), 143-148 (2017)

22. Y.A. Vasiliev, Digital Microfocus Radiography Technology In Assessing The Anatomical Structure Of Teeth (Moscow, Ph.D. Thesis, 2015)

23. A.D. Levitskaya, E.S. Syutkina, O.S. Gileva, S.V. Galkin, A.A. Efimov, Ya.V. Savitskii, Russian Journal of Biomechanics, 22(4), 425-441 (2018)

24. A.M. Kielbassa, U. Oeschger, J. Schulte-Monting, H. Meyer-Lueckel, J. Oral Rehabil., 32(2), 90-96 (2005). doi:10.1111/j.1365-2842.2004.01392.x

25. E.Z. Alsayed, I. Hariri, S. Nakashima, Y. Shimada, T.A. Bakhsh, J. Tagami, A. Sadr, Dental materials: official publication of the Academy of Dental Materials, 32(6), 807816 (2016). https://doi.org/10.1016/j.dental.2016.03.023 
26. R. Al-Obaidi, H. Salehi, A. Desoutter, L. Bonnet, P. Etienne, E. Terrer, B. Jacquot, B. Levallois, H. Tassery, F. Cuisinier, Scientific reports, 8(1), 4047 (2018). https://doi.org/10.1038/s41598-018-22459-7

27. D.J. White, Adv. Dent. Res., 9(3), 175-197 (1995). doi:10.1177/08959374950090030101

28. W. Buchalla, T. Imfeld, T. Attin, M.V. Swain, P.R. Schmidlin, Caries Res., 42(3), 157-163 (2008). doi:10.1159/000128559

29. J. Espigares, A. Sadr, H. Hamba, Y. Shimada, M. Otsuki, J. Tagami, Y. Sumi, J. Med. Imaging (Bellingham), 2(1), 014001 (2015). doi:10.1117/1.JMI.2.1.014001

30. H. Hamba, T. Nikaido, A. Sadr, S. Nakashima, J. Tagami, J. Dent. Res., 91(6), 586-591 (2012). doi:10.1177/0022034512444127 